\title{
Effectiveness of behavioural graded activity compared with physiotherapy treatment in chronic neck pain: design of a randomised clinical trial [ISRCTN88733332]
}

\author{
Frieke Vonk ${ }^{* 1}$, Arianne P Verhagen ${ }^{1}$, Mario Geilen ${ }^{2}$, Cees J Vos ${ }^{1}$ and \\ Bart W Koes ${ }^{1}$
}

Address: ${ }^{1}$ Department of General Practice, Erasmus MC, University Medical Centre Rotterdam, the Netherlands and ${ }^{2}$ Department of Rheumatic diseases and Chronic pain, Hoensbroek Rehabilitation Centre, the Netherlands

Email: Frieke Vonk* - f.vonk@erasmusmc.nl; Arianne P Verhagen - a.verhagen@erasmusmc.nl; Mario Geilen - m.geilen@srl.nl; Cees J Vos - c.vos@erasmusmc.nl; Bart W Koes - b.koes@erasmusmc.nl

* Corresponding author

Published: 06 October 2004

BMC Musculoskeletal Disorders 2004, 5:34 doi:10.1 | 86/| 47/-2474-5-34

This article is available from: http://www.biomedcentral.com/I47/-2474/5/34

(C) 2004 Vonk et al; licensee BioMed Central Ltd.

This is an open-access article distributed under the terms of the Creative Commons Attribution License (http://creativecommons.org/licenses/by/2.0), which permits unrestricted use, distribution, and reproduction in any medium, provided the original work is properly cited.
Received: 06 August 2004

Accepted: 06 October 2004

\begin{abstract}
Background: Chronic neck pain is a common complaint in the Netherlands with a point prevalence of $14.3 \%$. Patients with chronic neck pain are often referred to a physiotherapist and, although many treatments are available, it remains unclear which type of treatment is to be preferred.

The objective of this article is to present the design of a randomised clinical trial, Ephysion, which examines the clinical and cost effectiveness of behavioural graded activity compared with a physiotherapy treatment for patients with chronic non-specific neck pain.

Methods: Eligible patients with non-specific neck pain persisting longer than 3 months will be randomly allocated to either the behavioural graded activity programme or to the physiotherapy treatment. The graded activity programme is based on an operant approach, which uses a timecontingent method to increase the patient's activity level. This treatment is compared with physiotherapy treatment using a pain-contingent method.

Primary treatment outcome is the patient's global perceived effect concerning recovery from the complaint. Global perceived effect on daily functioning is also explored as primary outcome to establish the impact of treatment on daily activity. Direct and indirect costs will also be assessed. Secondary outcomes include the patient's main complaints, pain intensity, medical consumption, functional status, quality of life, and psychological variables. Recruitment of patients will take place up to the end of the year 2004 and follow-up measurement will continue until end 2005.
\end{abstract}

\section{Background}

\section{Prevalence and incidence}

Neck pain is a common complaint that causes substantial morbidity in western countries with a reported prevalence ranging from 9.5 to $22 \%[1,2]$. Of all musculoskeletal pains in the Netherlands, neck pain is one of the three most reported with a point prevalence of $21 \%$; it is more often reported by women than men [3]. In 1996 total 
related costs were estimated to be US $\$ 686.2$ million, which is about $1 \%$ of the total Dutch health care expenditures [4]. Most neck complaints are continuous or recurrent [3]. When the neck pain persists for more than 3 months it is defined as chronic, and the related prevalence is $14.3 \%[3,5]$. Although the prevalence of neck pain is stable over different age groups, the incidence of chronic neck pain increases with age $[3,6]$.

There are many potential causes of neck pain, but mostly no specific underlying pathology is found so that it is designated as non-specific [7]. Although not a life- threatening disease, neck pain can negatively affect patients' quality of life, cause pain and stiffness, and may result in substantial medical consumption, absenteeism and disability $[4,8]$.

In the Netherlands, patients with neck pain are often referred for physiotherapy. Moreover, physiotherapy accounted for $84 \%$ of the total direct medical neck pain costs in 1996 [4]. Although physiotherapists can apply various treatments, no formal guidelines are yet available.

\section{Treatment models}

Two treatment models have been described in the literature, both of which are applicable within the field of physiotherapy. The first, a biomedical model, considers pain to be a sign of physiological damages and treatment according to this model aims to remove the pathologic condition so that the pain will no longer occur $[9,10]$. Moreover, treatment is guided by the amount of pain a patient experiences, leading to a pain-contingent approach [11]. According to the second, a biopsychosocial model, pain is not necessarily caused by underlying pathology or impairment but can persist long after the initial pathology has healed; psychological and social factors may be important in the development and maintenance of complaints [12,13]. According to the principles of this biopsychosocial model, behavioural therapies assume that maladaptive behaviours are learned and, therefore, can be modified through new learning experiences $[10,14]$. Three different approaches are known: respondent, operant and, cognitive behavioural therapy $[9,15,16]$. The present study mainly employs an operant behavioural approach, as described by Fordyce and applied by Lindström et al $[11,17]$. According to this approach, the treatment focuses on decreasing pain behaviour (operants) and increasing healthy behaviour, and consists of behavioural graded activity on a time-contingent basis $[11,18]$.

\section{Available evidence}

Many conservative physiotherapeutic treatments are available for treating neck pain, but there is insufficient evidence to allow to conclude that one type of treatment is more effective then others $[19,20]$.
In a review on chronic pain, operant behavioural therapy was found to be beneficial to waiting list control groups on outcomes such as pain experience, mood effect other than depression, social role, and for the expression of pain behaviour [21]. Compared to other treatments, operant behavioural therapy is only beneficial for the expression of pain behaviour and role functioning [21]. Another review showed little evidence that biopsychosocial multidisciplinary rehabilitation is more effective than other rehabilitation methods for neck and shoulder pain, but the authors found only two relevant studies that satisfied the criteria for their review [22]. When examining the effectiveness of behavioural treatment for chronic pain another difficulty is that no standard protocol exists for the application of these treatments. As a result, a wide range of techniques described in the literature has been labelled as behavioural [23].

In summary, it remains unclear which type of conservative, including behavioural, treatment is to be preferred in the management of chronic neck pain. Therefore, this study, Ephysion (Effectiveness physiotherapy in neck pain), aims to evaluate the clinical and cost effectiveness of an operant behavioural programme (i.e. behavioural graded activity) compared with a physiotherapy treatment in patients with chronic non-specific neck pain. In addition, we aim to identify subgroups of patients who benefit most from one of the two treatments, and to identify the most important determinants for recovery from chronic non-specific neck pain.

\section{Why a design article}

Because a biased study design can produce incorrect conclusions, the design of a trial should be carefully examined before adopting its conclusions [24]. A design article allows to examine the design objectively without being influenced by the study results, to check any resulting articles for protocol deviations, and may also reduce the temptation to search for associations during data analysis rather then presenting hypotheses in advance [25]. Further, a published protocol informs others about which studies are in process thus reducing duplication of research effort [25]. Finally, a design article prevents publication bias in the case that future articles are not published, because study results can be retrieved from the author and the study can therefore still be included in future reviews $[25,26]$.

\section{Methods \\ Study design}

A randomised clinical trial (RCT) has been designed to assess the effectiveness of behavioural graded activity compared with physiotherapy treatment in patients with chronic non-specific neck pain. The study design has been approved by the Medical Ethics Technical Commission of 
the Erasmus MC, University Medical Centre in Rotterdam and is in compliance with the Helsinki Declaration.

\section{Selection of patients and informed consent}

Forty general practitioners (GP) in region West Brabant in the Netherlands will select the patients. Patients are eligible if they are aged between 18 and 70 years old, have suffered from neck pain for over three months, and have an adequate knowledge of the Dutch language. Excluded are patients diagnosed with a specific disorder (e.g. a slipped disc, a tumour or a lesion in the cervical spine), those who have had physical/manual therapy during the previous six months, those with a chronic disease (e.g. rheumatoid arthritis or coronary artery disease), or those who have to undergo surgery in the near future. Eligible patients will receive an information leaflet from their GP and the GP then informs the research department.

Thereafter, the research assistant contacts the patient, provides additional information about the implications of participation, re-checks the eligibility of the patient, and completes the informed consent procedure.

\section{Sample size}

The sample size for this study is calculated according to the global perceived effect (GPE). Based on previous studies, a 20\% difference in GPE is expected after completion of either treatment ( 9 weeks) and is considered to be clinically relevant; 160 patients are needed to detect this difference. In this calculation a power $(1-\beta)$ of $80 \%$ is taken into account. Thus, the inclusion of 80 patients per treatment group is planned.

\section{Randomisation}

An independent examiner using a computer-generated randomisation schema performs randomisation. To prevent unequal distribution, patients are pre-stratified based on three important prognostic factors: gender, age and the severity of the complaint, which are recorded at baseline [27]. Further, unequal group sizes are prevented by using a 6-block randomisation that equalizes allocation to the two treatment groups per stratum after every sixth patient [28]. After randomisation, patients choose a physiotherapist within the allocated treatment group. Then, to ensure that the treatment starts as soon as possible, the research assistant makes the first appointment for treatment.

\section{Blinding}

Patients are told to receive physiotherapy but are blinded to allocation of the two treatments; the content of the treatments is not described in the information leaflet. This enhances the quality of the study, because the patients themselves measure the effect of treatment. GPs are also blinded for allocation to prevent accidentally informing the patients of the allocated treatment. The physiothera- pists are not blinded for allocation, but the physiotherapists from each treatment group are kept strictly separate and are not involved in the outcome measurement. Finally, the primary investigator is blinded for patients' allocation but the research assistant is not; neither is involved in the outcome measurement.

\section{Physiotherapists and Interventions}

After receiving written information, 34 physiotherapists in region West Brabant will participate in either the physiotherapy treatment (PT) or the graded activity programme (GAP). To optimise the contrast between the two treatments, both groups are strictly separated throughout the study. The PT group consists of 16 physiotherapists and the GAP group of 18 physiotherapists. The PT physiotherapists participate in a meeting to standardize the physiotherapy treatment. The GAP physiotherapists are instructed on the behavioural graded activity approach during a two-day theoretical and practical training course.

Both interventions are performed in an outpatient setting. A maximum of 18 treatments per patient is set and each treatment takes about 30 minutes, which is in accordance with medical insurance policy in the Netherlands. Before treatment starts, physiotherapists receive a completed questionnaire about the patient's main complaints [29]; this questionnaire reveals the three daily activities which are considered the most important complaints to the patient. Physiotherapists can use these three activities in the process of formulating the patient's primary therapy aim. In both treatments, the physiotherapist starts with a physical examination of the patient and an anamnesis. Then an individually tailored program will be applied and the process recorded after each treatment session using a specially designed form.

\section{The physiotherapy treatment}

The content of the physiotherapy treatment is decided by consensus among the participating PT physiotherapists. Treatment is according to a biomedical model, which implies guidance based on the amount and severity of pain that the patient experiences.

By consensus, the physiotherapy treatment is divided into the patient's primary therapy aim, three general treatment goals, and several techniques to attain those goals. The primary therapy aim is defined as the result the patient wants to achieve by the end of therapy. A general treatment goal is a goal for each single treatment and could, therefore, differ per treatment session. Table 1 shows the three general treatment goals, together with the techniques physiotherapists can choose to attain them. In daily practice a broad spectrum of treatment techniques are available, but in this study the techniques to be used consist of physiotherapy techniques with a strong focus 
Table I: Treatment goals and techniques that can be used to reach the primary treatment aim.

\begin{tabular}{ll}
\hline Treatment goals & Techniques \\
\hline relaxation and preperation for exercise & - Massage \\
& - Relaxation exercise \\
& - Thoracal treatment up to thoracal 9. \\
& - Localized 3-d mobilization within physiological boundary of the joint capsule \\
& - Mobilization in al directions within physiological boundaries. \\
& - Traction within physiological boundaries. \\
& - Techniques of Mulliken excluding manipulation \\
& - Techniques of McKenzie excluding manipulation \\
& Can take place at the same time as the first treatment target. Education includes patient reassurance; \\
& explanation of (physiological) load and capability of carrying a load; and encouragement of physical activity \\
Education & - Passive exercise, guided active exercise, and active exercise \\
& - Exercise at the physiotherapist \\
& - Assign homework
\end{tabular}

on exercises. Moreover, manipulative techniques, acupuncture and other (alternative) techniques are excluded, as are physiotherapeutic applications such as ultrasound or diathermy.

\section{Behavioural graded activity}

An operant approach was the basis of the behavioural graded activity programme as used in this study. The treatment is according to a biopsychosocial model, which implies that it is guided by the patients' functional abilities and that time-contingent methods are used to increase the activity level of the patient [11]. The behavioural graded activity programme has three phases; a baseline phase, a treatment phase, and a generalization phase. These phases are not bound to strict time limits but can gradually merge into each other.

Before starting the baseline phase, the treatment vision and the patient's ideas about pain and its causes are discussed. The development and maintenance of pain will be explained and patients are reassured that it is safe to move and to increase their level of activity $[11,13,30]$. Both are explained by means of a pain model, which has been derived from the fear-avoiding-model of Vlaeyen et al. [13]. Thereafter primary therapy aims are formulated based on the patient's main complaints, which are described as three daily activities and were revealed in the baseline questionnaire. For each of these activities, a baseline level of intensity is determined based on a pain-contingent measure. This means that patients perform each activity at least three times, each time until they have to stop because of their pain. Afterwards, patient and physiotherapist together set a start quota and time-contingent treatment quotas for each activity. The quotas will be based on the patient's mean baseline scores, primary therapy aims [17], and on the behaviour that can be derived from the baseline measure. If necessary, facilitating disorder-oriented exercises can be added to the treatment as preparation for the activities that were pointed out as main complaints. The same approach as used for the main complaint is used for these exercises.

During the treatment phase, patients systematically increase the time-contingent quotas to enable them to reach their personal aims within a pre-set therapy time period. To ensure a successful experience during the first exercise, the start quota is below the mean baseline score. The pre-set exercise quotas have to be strictly followed; neither over-performance nor under-performance is allowed. During this phase the patient has to practice at home and document every activity or exercise on a performance chart. These charts will be discussed in the following treatment session and achievements will be reinforced while disregarding pain behaviours. Positive reinforcements of healthy behaviour and the patient's experiences of success are considered to be important to enhance the patient's motivations.

The generalization phase takes place at the end of the treatment phase. In this phase generalization of learned behaviour and management of relapses will be discussed.

\section{Outcome measurement}

Baseline questionnaires are sent after inclusion, which is as soon as possible after patients have consulted their GP. Outcome of intervention will be assessed at 4 and 9 weeks after randomisation; however, if the treatment is not finished at 9 weeks, the patients will receive an additional questionnaire (Ts) after finishing the treatment. Followup assessments are planned at 26 and 52 weeks after randomisation. 
Table 2: Overview of variables measured in this study

\begin{tabular}{|c|c|c|c|c|c|c|c|}
\hline \multirow[t]{2}{*}{ Variable } & \multicolumn{6}{|c|}{ Time Measured } & \multirow[t]{2}{*}{ Range of unit } \\
\hline & To & T4 & T9 & Ts & T26 & T52 & \\
\hline Inclusion and exclusion variables & $x$ & & & & & & \\
\hline Demographic variables & $x$ & & & & & & \\
\hline \multicolumn{8}{|l|}{ Baseline variables } \\
\hline Specific complaint characteristics & $x$ & & & & & & \\
\hline $\begin{array}{l}\text { Experience of the neck complaint and functioning in } \\
\text { daily activities }\end{array}$ & $x$ & & & & & & I-7 (Likert scale) \\
\hline Co-morbidity & $x$ & & & & & & \\
\hline Additional complaints & $\mathbf{x}$ & & & & & & \\
\hline \multicolumn{8}{|l|}{ Primary outcome } \\
\hline $\begin{array}{l}\text { 'Global perceived effect' (neck complaint and } \\
\text { functioning in daily activities) [3I] }\end{array}$ & & $x$ & $x$ & $x$ & $x$ & $x$ & I-7 (Likert scale) \\
\hline \multicolumn{8}{|l|}{ Secondary outcomes } \\
\hline Main complaint [29] & $x$ & $x$ & $x$ & $x$ & $x$ & $x$ & 0-10 (Likert scale) \\
\hline Pain (VAS) [3I] & $x$ & $x$ & $x$ & $x$ & $x$ & $x$ & \\
\hline Medical consumption & $x$ & $x$ & $x$ & $x$ & $x$ & $x$ & Dose per day \\
\hline Coping with Multi-dimensional pain (MPI) Part I-II [32] & $x$ & & $x$ & & $x$ & $x$ & 0-6 (Likert scale) \\
\hline Activity (MPI, part III) & $x$ & $x$ & $x$ & $x$ & $x$ & $x$ & 0-6 (likert scale) \\
\hline Specific functional status (NDI) [33] & $x$ & $x$ & $\mathbf{x}$ & $x$ & $x$ & $x$ & \\
\hline Quality of life (SF-36) $[34,35]$ & $x$ & & $x$ & & $x$ & $x$ & \\
\hline$(E Q-5 d)[35,36]$ & $x$ & $x$ & $x$ & $x$ & $x$ & $x$ & \\
\hline Work activities & $x$ & & $x$ & & $x$ & $x$ & Hours/week \\
\hline Satisfaction about treatment & & $x$ & $x$ & $x$ & $x$ & & I-5 (Likert scale) \\
\hline Compliance with treatment exercise & & $x$ & $x$ & $x$ & $x$ & $x$ & Number and time per week \\
\hline Additional treatments & & $x$ & $x$ & $x$ & $x$ & $x$ & Discipline and number of treatments \\
\hline Side-effects & & $x$ & $x$ & $x$ & $x$ & $x$ & Yes - No and any additional elucidation \\
\hline \multicolumn{8}{|l|}{ Psychological (prognostic) variables } \\
\hline Fear of movement (TSK) [37] & $x$ & & & & $x$ & $x$ & I-4 (likert scale) \\
\hline Catastrophizing (PCS) [38] & $x$ & & $x$ & & $x$ & & I-5 (likert scale) \\
\hline Depression (CES-D) [39] & $x$ & & $x$ & & $x$ & & I-4 (likert scale) \\
\hline Self-efficacy (PSEQ) [40] & $x$ & $x$ & $x$ & $x$ & $x$ & $x$ & $10-100 \%$ (very unsure - very sure) \\
\hline Stages of change (PSOCQ) [4I] & $x$ & & $x$ & & & & I-5 (likert scale) \\
\hline
\end{tabular}

Note: $T_{0}=$ baseline measurement, $T_{4}, T_{9},\left(T_{S}\right), T_{26}, T_{52}$ are follow-up measurements at 4, 9, 26 and 52 weeks, respectively, after randomisation. $T s$ was received at the end of treatment, when treatment lasted longer than 9 weeks. MPI, Multidimensional Pain inventory; NDI, Neck Disability Index; SF-36, Short Form 36; EQ-5d, Euroquol 5-Dutch language version; TSK, Tampa Scale for Kinesiophobia; PCS, Pain Catastrophizing Scale; CES-D, Centre for Epidemiologic Studies - Depression; PSEQ, Pain Self-Efficacy Scale; PSOCQ, Pain Stages Of Change Questionnaire.

All outcome measures are reported by means of mailed questionnaires. Table 2 presents the outcome variables, the instruments used and the moments at which they are measured.

Primary treatment outcome of this study is the global perceived effect, which is used to assess recovery from the complaint [31]. In addition, the global perceived effect in daily functioning was explored in order to also establish impact of treatment on daily activity. Both treatment outcomes (recovery of complaint and functioning in daily activity), are assessed on a 7-point Likert-scale, ranging from completely recovered (1) to worse than ever (7).
Costs are measured using a combination of questionnaires to collect data on direct medical costs (e.g. the amount of received treatment and additional therapy received), and indirect costs due to sick leave and disability.

Secondary outcome measures include main complaints, pain intensity, medical consumption, coping, functional status, quality of life, and psychological variables. Prognostic factors are measured including demographic variables, the baseline variables and the psychological variables (table 2). 


\section{Analyses}

Descriptive statistics will be used to examine comparability of baseline data between PT and GAP, and to check if randomisation was successful. Before this analysis, decisions about differences considered to be clinically relevant are made and, if necessary, adjustment will be made for these differences in multivariate analysis. Further, all outcome data will be screened for normality and, if necessary, logarithmic transformations or non-parametric methods of analysis will be applied.

The first aim is to evaluate the clinical and cost effectiveness of GAP compared to PT. Clinical effectiveness will be examined with a Student's t-test (continuous), a Chisquare test (dichotomised) or a Wilcoxon test (not normally distributed) according to the intention-to-treat principle. This means that patients will be analysed in the treatment group to which they are randomly allocated. For missing data, imputation techniques will be used. When the dropout rate is $10 \%$ or more, or loss to followup is $20 \%$ or more, per-protocol analysis will be performed. The results on primary outcome will be dichotomised into improved versus not improved. Improved implies completely recovered and much improved, whereas not recovered implies slightly improved, not changed, slightly worsened, much worsened, and worse than ever [31].

Cost effectiveness will be calculated from a societal perspective. Costs (direct as well as indirect) will be related to the treatment effects, based on the primary outcome measure, by calculating cost-effectiveness ratios.

The second aim is to identify subgroups of patients that benefit most from one of the two treatments. The following subgroups will be investigated: duration and severity of the complaint, depression, and fear of movement.

The third aim is to identify important variables for recovery. For this purpose multivariate analysis will be performed to investigate the influence of prognostic variables and patient characteristics on the outcome. Separate analyses will be conducted to investigate prognostic factors for short-term (3 months) and long-term (12 months) recovery.

\section{Discussion}

This study is designed to evaluate the clinical and cost effectiveness of a behavioural graded activity programme compared with a physiotherapy treatment in patients with chronic non-specific neck pain. Since physiotherapists perform both treatments in this study, contrast between the two treatments is a very important issue. There are contrasts both in the composition of the treatment and the way the physiotherapists approach the patient. With regard to the composition, the graded activity programme (GAP) starts with a systematically performed baseline measurement; this is in contrast to the physiotherapy treatment (PT), where treatment is based on history taking and physical examination. In GAP quotas are set based on the patient's behaviour, whereas in PT they are set based on pain levels and training principles. After quotas are set GAP uses a time-contingent treatment approach, which involves a pre-set systematic increase in activities. In contrast, PT uses a pain-contingent approach, which means that treatment is adapted to the patient's reaction to previous treatment sessions. Furthermore, GAP uses a handsoff approach, whereas PT may contain hands-on techniques, such as massage, traction etc (Table 1).

This study addresses an important question because chronic neck pain is a common complaint and it remains unclear which type of physiotherapeutic treatment is most effective. Recruitment of patients will take place until up to the end of 2004; follow-up measurement will continue up to end 2005.

\section{Competing interests}

The authors declare that they have no competing interests.

\section{Authors' contributions}

APV and BWK conceived the study, developed the design of the randomised clinical trial and participated in writing the article. MG is an expert in the field of graded activity and contributed to the content of the article. CJV advised on the content of the article. FV conducts the research, participated in the completion of the study design and wrote the article. All authors have read and approved the final manuscript.

\section{References}

I. Wolsko PM, Eisenberg DM, Davis RB, Kessler R, Phillips RS: Patterns and perceptions of care for treatment of back and neck pain: results of a national survey. Spine 2003, 28:292-7; discussion 298.

2. Andersson HI, Ejlertsson G, Leden I, Rosenberg C: Chronic pain in a geographically defined general population: studies of differences in age, gender, social class, and pain localization. Clin J Pain 1993, 9:174-182.

3. Picavet HS, Schouten JS: Musculoskeletal pain in the Netherlands: prevalences, consequences and risk groups, the DMC(3)-study. Pain 2003, I02:167-I78.

4. Borghouts JA, Koes BW, Vondeling H, Bouter LM: Cost-of-illness of neck pain in The Netherlands in 1996. Pain 1999, 80:629-636.

5. IASP: Classification of chronic pain. Descriptions of chronic pain syndromes and definitions of pain terms. Prepared by the International Association for the Study of Pain, Subcommittee on Taxonomy. Pain Suppl 1986, 3:SI-226.

6. Cassou B, Derriennic F, Monfort C, Norton J, Touranchet A: Chronic neck and shoulder pain, age, and working conditions: longitudinal results from a large random sample in France. Occup Environ Med 2002, 59:537-544.

7. Bogduk N: Neck pain. Aust Fam Physician 1984, 13:26-30.

8. Korthals-de Bos IB, Hoving JL, van Tulder MW, Rutten-van Molken MP, Ader HJ, de Vet HC, Koes BW, Vondeling H, Bouter LM: Cost effectiveness of physiotherapy, manual therapy, and general practitioner care for neck pain: economic evaluation alongside a randomised controlled trial. BMJ 2003, 326:9l I. 
9. Turk DC, Flor $\mathrm{H}$ : Etiological theories and treatments for chronic back pain. II. Psychological models and interventions. Pain 1984, 19:209-233.

10. Fordyce WE, Fowler R. S., Jr., Lehmann JF, Delateur BJ, Sand PL, Trieschmann RB: Operant conditioning in the treatment of chronic pain. Arch Phys Med Rehabil 1973, 54:399-408.

II. Lindstrom I, Ohlund C, Eek C, Wallin L, Peterson LE, Fordyce WE Nachemson AL: The effect of graded activity on patients with subacute low back pain: a randomized prospective clinical study with an operant-conditioning behavioral approach. Phys Ther 1992, 72:279-90; discussion 291-3.

12. Waddell G: 1987 Volvo award in clinical sciences. A new clinical model for the treatment of low-back pain. Spine 1987, 1 2:632-644.

13. Vlaeyen JW, Kole-Snijders AM, Boeren RG, van Eek H: Fear of movement/(re)injury in chronic low back pain and its relation to behavioral performance. Pain 1995, 62:363-372.

14. Linton SJ: Chronic back pain: integrating psychological and physical therapy--an overview. Behav Med 1994, 20:101-104.

15. Vlaeyen J. W.S., Kole-Snijders, A.M.J., van Eek, H., Heuts, P.H.T.G.: Gedragstherapeutische toepassing bij (chronische) lage rugpijn. Medische Psychologie 1995, maart: 157-164.

16. Gatchel Robert J., Turk Dennis C.: Psychological Approaches to Pain Management; A Practitioner's Handbook. Edited by: Robert J Gatchel and Dennis C Turk. New York London, The Guilford Press; 1996.

17. Fordyce WE: Behavioral Methods for Chronic Pain and IIIness. St. Louis, C. V. Mosby; 1976.

18. Vlaeyen JW, Haazen IW, Schuerman JA, Kole-Snijders AM, van Eek $\mathrm{H}$ : Behavioural rehabilitation of chronic low back pain: comparison of an operant treatment, an operant-cognitive treatment and an operant-respondent treatment. $\mathrm{Br} J$ Clin Psychol 1995, 34:95-II8.

19. Aker Peter D, Gross Anita R, Goldsmith Charles H, Peloso Paul: Conservative management of mechanical neck pain: systematic overview and meta-analysis. BM] 1996, 3 13:129|-I 296.

20. Hoving JL, Gross AR, Gasner D, Kay T, Kennedy C, Hondras MA Haines T, Bouter LM: A critical appraisal of review articles on the effectiveness of conservative treatment for neck pain. Spine 200I, 26:196-205.

21. Morley S, Eccleston C, Williams A: Systematic review and metaanalysis of randomized controlled trials of cognitive behaviour therapy and behaviour therapy for chronic pain in adults, excluding headache. Pain 1999, 80: I-13.

22. Karjalainen $K$, Malmivaara $A$, van Tulder $M$, Roine $R$, Jauhiainen $M$ Hurri H, Koes B: Multidisciplinary biopsychosocial rehabilitation for neck and shoulder pain among working age adults: a systematic review within the framework of the Cochrane Collaboration Back Review Group. Spine 2001, 26:174-18I.

23. Turner JA, Clancy S: Comparison of operant behavioral and cognitive-behavioral group treatment for chronic low back pain. J Consult Clin Psychol 1988, 56:261-266.

24. Moore RA: Pain and systematic reviews. Acta Anaestesiol Scand 200I, 45: II36-II39.

25. Godlee F: Publishing study protocols: making them visible will improve registration, reporting and recruitment. $B M C$ News and Views 200I, 2.

26. Ostelo RW, Koke AJ, Beurskens AJ, de Vet HC, Kerckhoffs MR, Vlaeyen JW, Wolters PM, Berfelo MW, van den Brandt PA: Behavioral-graded activity compared with usual care after firsttime disk surgery: considerations of the design of a randomized clinical trial. J Manipulative Physiol Ther 2000, 23:3 | 2-319.

27. Borghouts JA, Koes BW, Bouter LM: The clinical course and prognostic factors of non-specific neck pain: a systematic review. Pain 1998, 77:1-13

28. Roberts $C$, Torgerson $D$ : Randomisation methods in controlled trials. $B M]$ 1998, 317:| I30I

29. Beurskens AJ, de Vet HC, Koke AJ, Lindeman E, van der Heijden GJ, Regtop W, Knipschild PG: A patient-specific approach for measuring functional status in low back pain. I Manipulative Physio Ther 1999, 22: 144-148.

30. Vlaeyen JWS: Chronic low back pain: assessment and treatment from a behavioral rehabilitation perspective. Amsterdam, Swets en Zeitlinger; 1991.
3I. Beurskens AJ, de Vet HC, Koke A): Responsiveness of functional status in low back pain: a comparison of different instruments. Pain 1996, 65:71-76.

32. Lousberg R, Van Breukelen G], Groenman NH, Schmidt AJ, Arntz A Winter FA: Psychometric properties of the Multidimensional Pain Inventory, Dutch language version (MPI-DLV). Behav Res Ther 1999, 37:167-| 82.

33. Wheeler AH, Goolkasian P, Baird AC, Darden B. V., 2nd: Development of the Neck Pain and Disability Scale. Item analysis, face, and criterion-related validity. Spine 1999, 24: I 290-I 294.

34. Keller SD, Ware J. E., Jr., Bentler PM, Aaronson NK, Alonso J, Apolone G, Bjorner JB, Brazier J, Bullinger M, Kaasa S, Leplege A, Sullivan M, Gandek B: Use of structural equation modeling to test the construct validity of the SF-36 Health Survey in ten countries: results from the IQOLA Project. International Quality of Life Assessment. J Clin Epidemiol 1998, 5 I: I I 79- I 188.

35. Essink-Bot ML, Krabbe PF, Bonsel G], Aaronson NK: An empirical comparison of four generic health status measures. The Nottingham Health Profile, the Medical Outcomes Study 36item Short-Form Health Survey, the COOP/WONCA charts, and the EuroQol instrument. Med Care 1997 35:522-537.

36. Van Agt HM, Essink-Bot ML, Krabbe PF, Bonsel G]: Test-retest reliability of health state valuations collected with the EuroQol questionnaire. Soc Sci Med 1994, 39: I537-I 544.

37. Swinkels-Meewisse EJ, Swinkels RA, Verbeek AL, Vlaeyen JW, Oostendorp RA: Psychometric properties of the Tampa Scale for kinesiophobia and the fear-avoidance beliefs questionnaire in acute low back pain. Man Ther 2003, 8:29-36.

38. Osman A, Barrios FX, Gutierrez PM, Kopper BA, Merrifield T, Grittmann L: The Pain Catastrophizing Scale: further psychometric evaluation with adult samples. J Behav Med 2000, 23:35 I-365.

39. Bouma J, Ranchor AV, Sanderman R, Van Sonderen FLP.: Het meten van symptomen van depressie met de CES-D. Een handleiding. Groningen, Noordelijk Centrum voor Gezondheidsvraagstukken, Rijksuniversiteit Groningen; 1995:2-24.

40. Anderson KO, Dowds BN, Pelletz RE, Edwards WT, Peeters-Asdourian C: Development and initial validation of a scale to measure self-efficacy beliefs in patients with chronic pain. Pain 1995, 63:77-84.

4I. Kerns RD, Rosenberg R, Jamison RN, Caudill MA, Haythornthwaite J: Readiness to adopt a self-management approach to chronic pain: the Pain Stages of Change Questionnaire (PSOCQ). Pain 1997, 72:227-234

\section{Pre-publication history}

The pre-publication history for this paper can be accessed here:

http://www.biomedcentral.com/1471-2474/5/34/prepub

Publish with Biomed Central and every scientist can read your work free of charge

"BioMed Central will be the most significant development for disseminating the results of biomedical research in our lifetime. "

Sir Paul Nurse, Cancer Research UK

Your research papers will be:

- available free of charge to the entire biomedical community

- peer reviewed and published immediately upon acceptance

- cited in PubMed and archived on PubMed Central

- yours - you keep the copyright

Submit your manuscript here:

http://www.biomedcentral.com/info/publishing_adv.asp
BiolMedcentral 